\title{
Analysing the Contributions in Moment Equations of Reynolds Stress Models of Convection with Numerical Simulations
}

\author{
F. Kupka ${ }^{1}$, H. J. Muthsam ${ }^{2}$ \\ ${ }^{1}$ Max-Planck-Institute for Astrophysics, Karl-Schwarzschild Str. 1, D-85748 Garching, \\ Germany \\ email: fk@mpa-garching.mpg.de \\ ${ }^{2}$ Institute of Mathematics, University of Vienna, Nordbergstraße 15, A-1090 Vienna, Austria, \\ email: herbert.muthsam@univie.ac.at
}

\begin{abstract}
We discuss how 3D numerical simulations can be used to analyse the different contributions within dynamical equations of non-local Reynolds stress models of convection.
\end{abstract}

Keywords. Convection, turbulence, stars: interiors

\section{Models, numerical simulations, model cases, and discussion}

Reynolds stress models for turbulent convection require to solve dynamical equations for the ensemble averages of velocity and temperature fields and their lower order moments. Because of the non-linearity of the underlying hydrodynamical equations these equations are part of an unclosed hierarchy which requires additional assumptions (closure approximations) to formulate predictive models. In Kupka \& Muthsam (2007a-c) and Kupka (2007) a study of a set of moment equations proposed by Canuto \& Dubovikov (1998) (combined with results from Canuto 1992, 1993) was presented. They extended earlier tests by Kupka (1999), which had been based on 3D numerical simulations of compressible convection by Muthsam et al. $(1995,1999)$, to the case of deep convection zones. We present further results using a term-by-term analysis of individual contributions in the dynamical equations. The simulations are briefly described in Kupka \& Muthsam (2007a), and resolve all scales down to the dissipation range for a given, constant Prandtl number $\operatorname{Pr}$, assume idealised microphysics, a perfect gas with $\gamma=5 / 3$, prescribed radiative conductivities, and a cartesian geometry with a constant, downwards pointing gravitational acceleration. Horizontal boundary conditions are periodic, vertical ones are closed and stress-free with a constant energy flux imposed at the bottom and a constant temperature at the top. Radiative transfer is treated in the diffusion approximation. As in Kupka \& Muthsam (2007a) we consider a thin zone of inefficient convection embedded in stably stratified layers ('model $3 \mathrm{~J}$ ', $72 \times 50^{2}$ grid points, $\mathrm{Pr}=1$ ) along side simulations of a thicker zone with more efficient convection embedded in likewise manner ('model $155 X^{\prime}$ with $\operatorname{Pr}=0.1$ and a resolutions of $160 \times 140^{2}$ points, first component vertical). Solutions of Reynolds stress models are obtained with a modified version of the code of Kupka (1999). The model equations are those suggested in Canuto \& Dubovikov (1998) (CD98) with some extensions taken from Canuto $(1992,1993,1997)$ and Canuto et al. (2001). For the time scales $\tau_{\theta}$ and $\tau_{p \theta}$ the high Peclet number (Pe) limit of CD98 was taken, since their low Pe number limits are not applicable to moderate Prandtl numbers.

As shown in Fig. 1 for model '3J' the simulations at least broadly agree with the most complete model (compressibility terms and residuals are not shown, the unstable zone is 

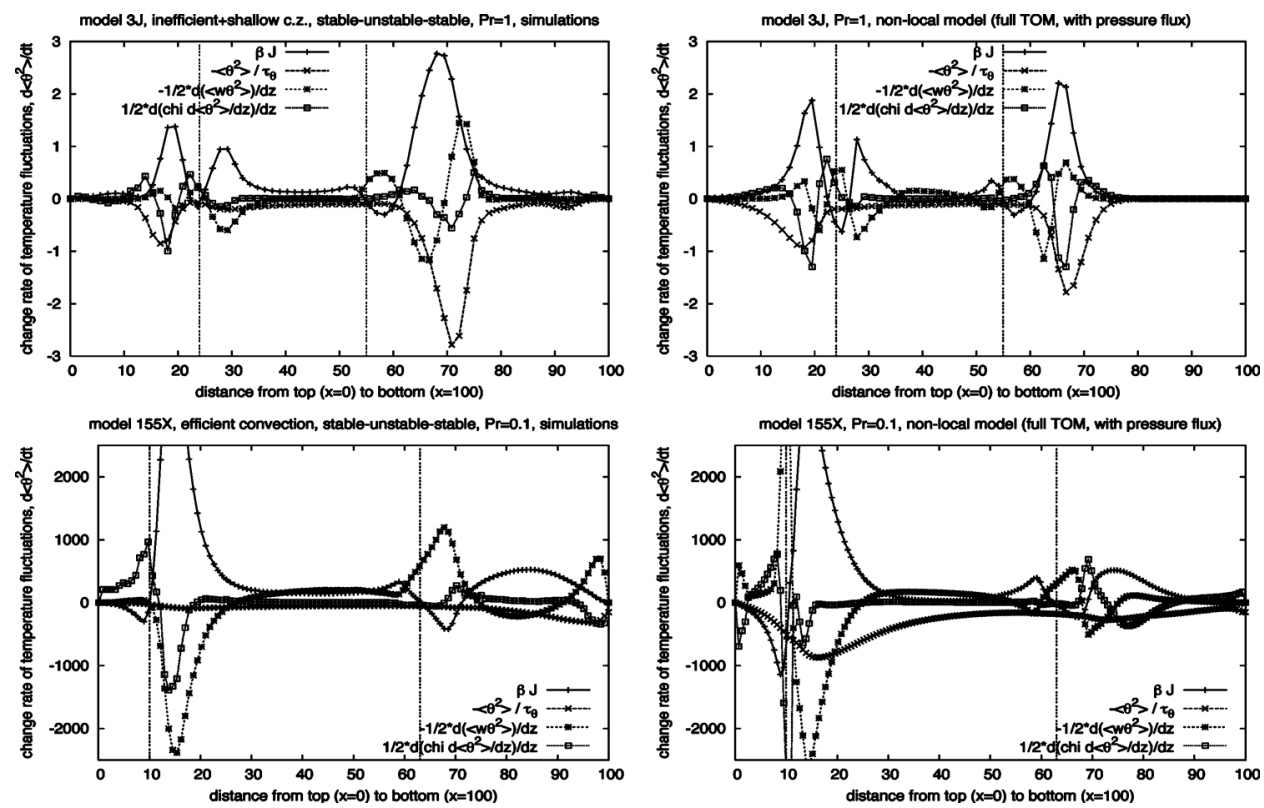

Figure 1. Model terms for the change rate of temperature fluctuations as found in simulations (left panels) and the model (right panels) for case ' $3 \mathrm{~J}$ ' (top row) and '155X' (bottom row).

located between the two vertical lines) on the overall shape and the contribution of the main terms in the dynamical equation for temperature fluctuations. Similar is found for the other equations of the model which helps to explain its success for shallow convection zones of A-type stars (Kupka \& Montgomery 2002). But for deep, efficient convection ('155X') the discrepancies are much larger, both in terms of the shape and the size of the individual terms of the dynamical equation investigated here.

\section{Acknowledgements}

The work of F. Kupka is supported by the DFG subproject KU 1954/3-1 of SPP 1276/1. H.J. Muthsam acknowledges support from FwF projects P17024 and P18224.

\section{References}

Canuto, V. M. 1992, ApJ 392, 218

Canuto, V. M. 1993, ApJ 416, 331

Canuto, V. M. 1997, ApJ 482, 827

Canuto, V. M. \& Dubovikov, M. S. 1998, ApJ 493, 834 (CD98)

Canuto, V. M., Cheng, Y., \& Howard, A. 2001, J. Atmos. Sci. 58, 1169

Kupka, F. 1999, ApJ (Letters) 526, L45

Kupka, F. 2007, in: F. Kupka, I. W. Roxburgh, \& K.L. Chan (eds.), Proc. IAU S 239, (Cambridge: Camb. Univ. Press), p. 92

Kupka, F. \& Montgomery, M. H. 2002, MNRAS 330, L6

Kupka, F. \& Muthsam, H. J. 2007a, in: F. Kupka, I. W. Roxburgh \& K. L. Chan (eds.), Proc. IAU S 239, (Cambridge: Camb. Univ. Press), p. 80

Kupka, F. \& Muthsam, H. J. 2007b, in: F. Kupka, I. W. Roxburgh \& K. L. Chan (eds.), Proc. IAU S 239, (Cambridge: Camb. Univ. Press), p. 83

Kupka, F. \& Muthsam, H. J. 2007c, in: F. Kupka, I. W. Roxburgh \& K. L. Chan (eds.), Proc. IAU S 239, (Cambridge: Camb. Univ. Press), p. 86

Muthsam, H. J., Göb, W., Kupka, F., Liebich, W., \& Zöchling, J. 1995, A\&GA 293, 127

Muthsam, H. J., Göb, W., Kupka, F., \& Liebich, W. 1999, New Astronomy 4, 405 